\title{
Land surface albedo from MSG/SEVIRI: retrieval method, validation, and application for weather forecast
}

\author{
Dominique Carrerr ${ }^{1}$,Bernhard Geigerr, ${ }^{1,2}$, Jean-Louis Roujean ${ }^{1}$, Olivier Hautecoeur ${ }^{1}$, Jure Cedilnik ${ }^{3}$, Jean-François \\ Mahfouf ${ }^{l}$, Catherine Meurey ${ }^{I}$, and Laurent Franchistéguy ${ }^{1,4}$ \\ 1. Météo-France, CNRM/GMME, 42 av. G. Coriolis, 31057 Toulouse, France \\ 2. ESA/ESAC, P.O Box 78, 28691 Villanueva de la Canada, Spain \\ 3. Slovenian Meteo Service, Environmental Agency of Slovenia, Vojkova 1b, SI-1000 Ljubljana, Slovenia \\ 4. now at Météo-France/Direction de la Climatologie, 42 avenue Gaspard Coriolis, 31057 Toulouse \\ Email: dominique.carrer@meteo.fr
}

\begin{abstract}
The European Meteorological Satellite Organization (EUMETSAT) maintains a number of decentralized processing centers dedicated to different scientific themes. The Portuguese Meteorological Institute hosts the Satellite Application Facility on Land Surface Analysis (LSA-SAF). The primary objective of the LSA-SAF is to provide added-value products for the meteorological and environmental science communities with main applications in the fields of climate modeling, environmental management, natural hazards management, and climate change detection. Since 2005 data from Meteosat Second Generation satellite are routinely processed in near real time by the LSA-SAF operational system in Lisbon. Presently, the delivered operational products comprise land surface albedo and temperature, shortwave and long-wave downwelling radiation fluxes, vegetation parameters and snow cover. After more than ten years (1999-2010) of research, development, and progressive operational activities, a summary of the surface albedo product characteristics and performances is presented. The relevance of LSA-SAF albedo product is analyzed through a weather forecast model (ALADIN) in order to account for the inter-annual spatial and temporal variability. Results clearly show a positive impact on the 12-hour forecast of $2 \mathrm{~m}$ temperatures.
\end{abstract}

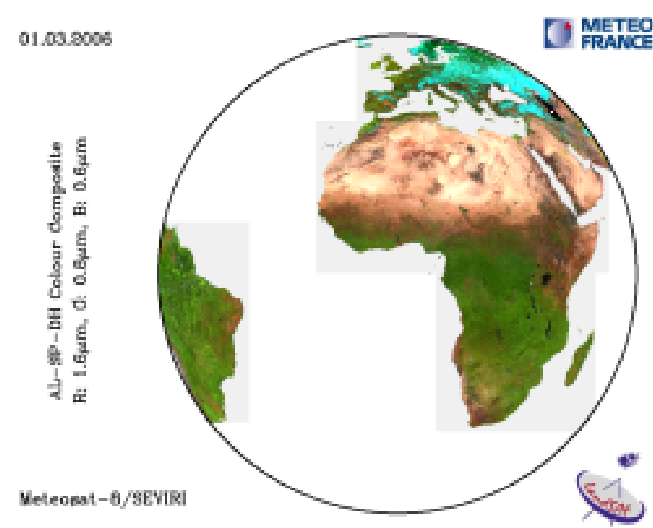

Figure 1: Colour composite product from the three spectral albedo estimates for the $1^{\text {ti }}$ of March 2006 over MSG disk.

\section{INTRODUCTION}

The Spinning Enhanced Visible and Infrared Imager (SEVIRI) radiometer embarked on the Meteosat Second Generation (MSG) platform encompasses unique spectral characteristics and accuracy, with a $3 \mathrm{~km}$ resolution (sampling distance) at nadir, 12 spectral channels, and an imaging repeat cycle of 15 minutes (e.g., [1]). The land surface albedo products is derived from the $0.6 \mu \mathrm{m}, 0.8 \mu \mathrm{m}$, and $1.6 \mu \mathrm{m}$ channels of the SEVIRI instrument (product examples are shown in Figure 1). Albedo maps are provided once a day based on the most recent cloud-free observations available. The albedo product is still "pre-operational" which means, according to the relevant EUMETSAT terminology, that it is "able to satisfy majority of applicable requirements" and has been considered "suitable for early distribution to the users with documented limitations".

The product files are generated in HDF5 format, and disseminated with a timeliness of 3 hours via the LSASAF website (http://landsaf.meteo.pt/) and by the EUMETSAT broadcast system EUMETCast. 


\section{SURFACE ALBEDO}

\subsection{Methodology}

The basic algorithm concept comprises an atmospheric correction scheme, the inversion of a linear semiempirical model of the bi-directional reflectance distribution function (BRDF), an angular integration of the latter to obtain spectral DHR and BHR (Directional and Bi-directional Hemispherical Reflectance) products, and the application of suitable conversion relationships to derive 3 broadband albedo estimates in the visible, near infrared and shortwave. The BRDF model inversion is performed each day based on the available set of clear-sky observations. In addition, constraints on the model parameters are taken into account in the inversion process. By specifying these constraints according to the previous model output in a recursive manner, a complete spatial coverage of the resulting albedo maps is achieved while at the same time preserving a high temporal resolution [2].

\subsection{Assessment}

The albedo product has been compared to the respective product derived from the MODIS (MODerate Resolution Imaging Spectroradiometer) instruments aboard NASA's Terra and Aqua platforms and to in-situ measurements over Europe and the Northern Africa region. We re-projected the $1 \mathrm{~km}$ resolution MODIS product to the MSG/SEVIRI grid. All MODIS pixels assigned to the near neighbour SEVIRI pixel were averaged to obtain albedo estimates to be compared with SEVIRI. The MODIS collection 4 product (V004) is delivered on a 16-day basis, retrieved at an 8-day frequency in latest version 5 (V005). In order to reproduce MODIS temporal characteristics the closest to SEVIRI products, the internal TOC (Top Of Canopy) reflectance files provided by the operational system were re-processed to generate daily albedo estimates, which were then averaged over the relevant MODIS period.

The comparative study with the MODIS albedo product shows a good consistency over mid-latitude regions for both LSA-SAF shortwave and near infrared broadband albedo estimates: biases are below 0.01 in absolute unit and $5 \%$ in relative unit while the standard deviation is in order of 0.02 [3]. The temporal evolution statistics of comparison from June 2005 to September 2006 over Europe is visualised in Figure 2. In relative units, none degradation of those results exists over bright surfaces (in North Africa). If MODIS is considered as a valid (unbiased) reference, a bias requirement of $10 \%$ can be considered as fulfilled for these LSA-SAF products except for pixels flagged as snow/ice covered. For snow/ice areas, the high frequency of LSA-SAF product takes advantage to track occasional events. However, more investigations are probably needed to estimate albedo more accurately over snow-covered surfaces classified as shrub land or forests (potential use of a specific snow/ice BRDF model).
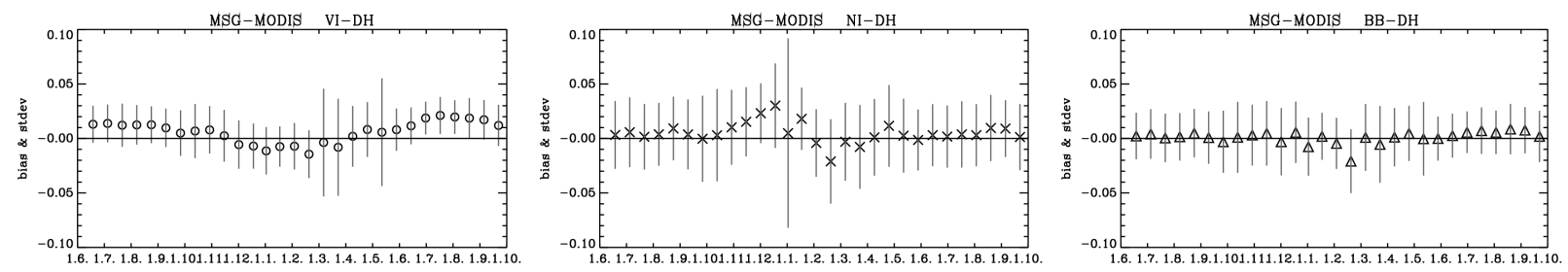

Figure 2: Time series of statistics over Europe of DHR albedo (in absolute units) from June 2005 to September 2006. The X-axis time label 1.6 refers to first of June. Left: Visible broadband. Middle: Near infrared broadband. Right: Shortwave broad-band. 

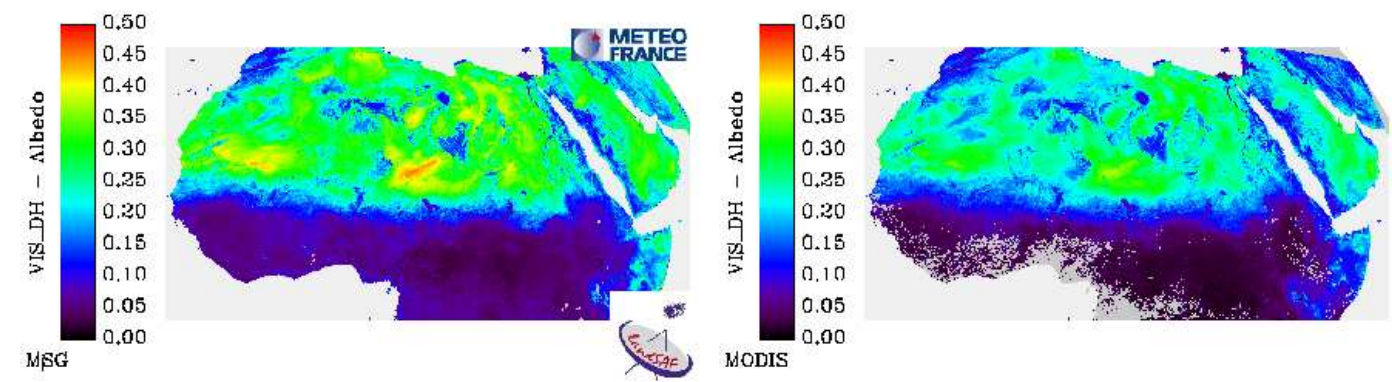

Figure 3: Comparison of visible broadband DHR albedo: LSA-SAF (left), and MODIS (right).

For the visible broadband albedo product the overestimation is beyond the specification (around 20\% in relative units). Figure 3 gives an example of the overestimation of VIS-DH MSG estimates in comparison to MODIS data for the period from July 12 of 2006 to July 27 of 2006 (MODIS period 193). This could be somewhat improved by revising the narrow- to broadband conversion and by the use of a better aerosol product for atmospheric correction. However, sensitivity studies show that a part of the discrepancy can be due to the use of different BRDF models. For suitable sites, comparison studies at the level of directional reflectances after atmospheric correction will be considered in a future. Detailed description is given in the Validation Report (LSA-SAF, 2007) available on the project website.

\subsection{Application for weather forecast}

Land surface schemes of NWP models use static albedo products over snow-free surfaces. An optimal analysis of LSA-SAF surface albedo was developed for snow-free pixels by combining a-priori information (climatology) with SEVIRI near real-time product. The surface analysis system is based on the use of Kalman filter. It was evaluated on the limited area weather forecast model ALADIN. Runs were performed from February 1st until end of July 2007, starting at 00UTC for up to +54 hours over an operational domain covering Eastern Europe where spatial resolution is around $9.5 \mathrm{~km}$.

Figure 4 shows difference for February 15, 2007 between the LSA-SAF and ALADIN albedo values and the associated impact on the forecast atmospheric temperature at 2 meters. This latter is enhanced where the LSA-SAF albedo is lower compared to ALADIN albedo. An absolute difference about $1 \mathrm{~K}$ is noticed over Pô region in Italy or Rhône valley in France. Statistical scores over a 6-month period show that ALADIN has a significant cold bias in winter during daytime, and that satellite data reduce it. It seems that the greatest impact on temperature scores corresponds to maximum downward solar radiation during daytime. 

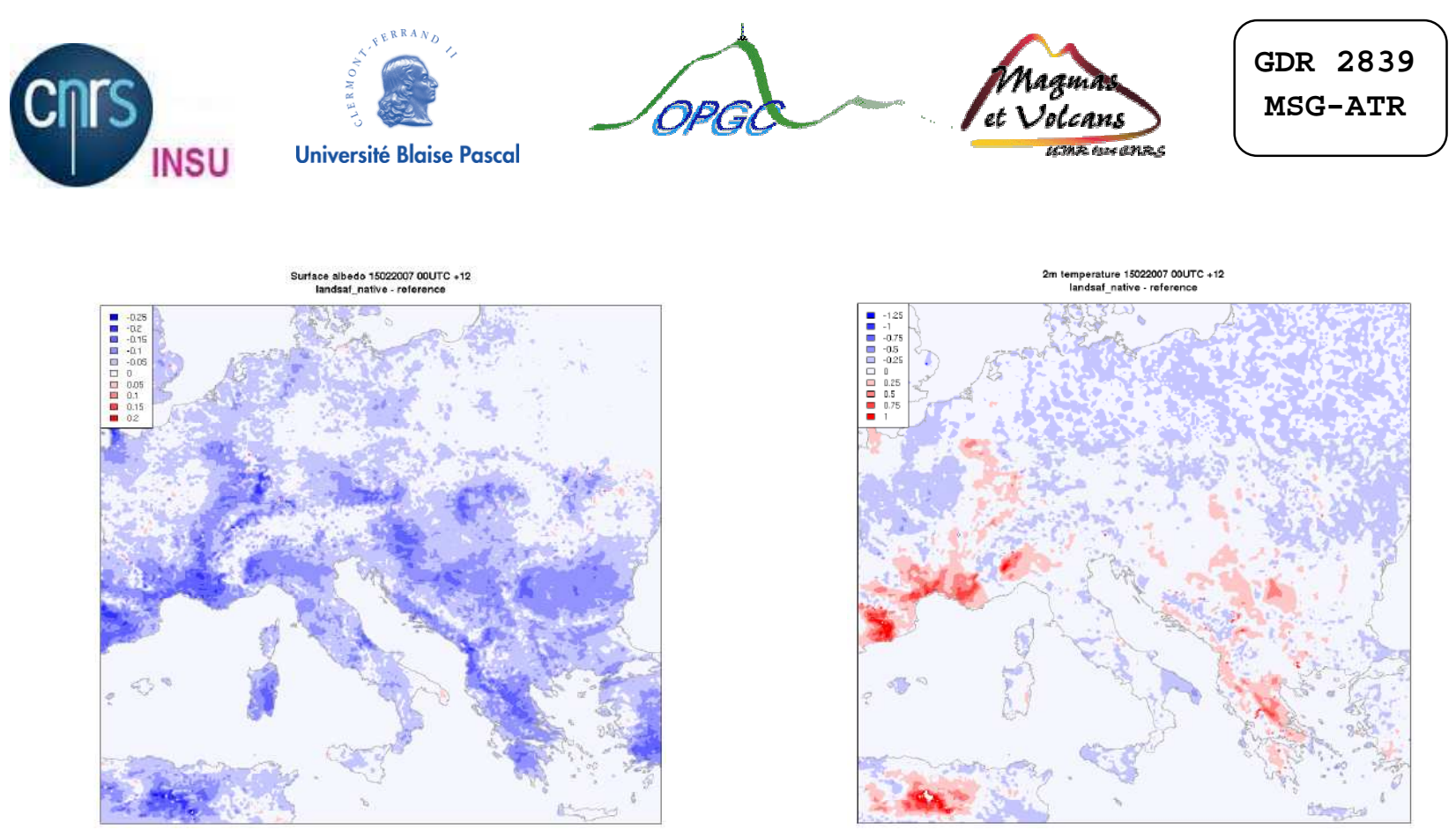

Figure 4: Difference in surface albedo between LSA-SAF and ALADIN (left). Difference in 2-meters temperature after 12 hours of integration from ALADIN runs using analyzed albedo (using LSA-SAF albedo) and the ALADIN albedo of reference. The date is February 15, 2007.

\section{PERSPECTIVES}

The reliability of the LSA-SAF albedo products has been verified through a direct assessment with in-situ observations and similar MODIS products. An indirect verification was done by using the surface albedo products in the weather forecast model ALADIN and considering the forecast near-surface temperature scores for diagnostic. Further improvement of the quality of the LSA-SAF albedo will arise from a better aerosol correction thanks to the recent development of an innovative method to derive a daily-based aerosol optical thickness (AOT).

At the end of the present CDOP phase (the Continuous Development and Operational Phase, 2007-2012) the LSA- SAF project will provide enhanced product generation by means of merging data between the polar satellite MetOp (Meteorological Operational, launched in October 2006) and the MSG-2 geostationary satellite data. The additional information will be particularly beneficial for high latitudes during winter and snow albedo in general. The LSA-SAF program provides a great opportunity to monitor and identify human-induced climate change as a consistent production of data sets is guaranteed until at least 2019 with the forthcoming MSG-3 mission.

\section{REFERENCES}

[1] Schmetz, J., P. Pili, S. Tjemkes, D. Just, J. Kerkman, S. Rota, and A. Ratier, “An introduction to Meteostat Second Generation (MSG)”, Bull. Amer. Meteor. Soc., 83, 977-992, 2002.

[2] Geiger, B., D. Carrer, L. Franchistéguy, J.L. Roujean, and C. Meurey, "Land Surface Albedo derived on a daily basis from Meteosat Second Generation Observations", IEEE Transactions on Geoscience and Remote Sensing, vol.46, no.11, pp.3841-3856, 2008.

[3] Carrer, D., J.L. Roujean, and C. Meurey, "Comparing operational MSG/SEVIRI land surface albedo products from Land SAF with ground measurements and MODIS", IEEE Transactions on Geoscience and Remote Sensing, doi:10.1109/TGRS.2009.2034530, in press. 